\title{
Long-Term Structural Modification of Water under Microwave Irradiation: Low-Frequency Raman Spectroscopic Measurements
}

\author{
A. V. Yakunov, M. M. Biliy, and A. P. Naumenko \\ Taras Shevchenko National University of Kyiv, 64/13 Volodymyrska Street, Kyiv 01601, Ukraine \\ Correspondence should be addressed to A. P. Naumenko; apn@voliacable.com
}

Received 15 November 2016; Revised 24 January 2017; Accepted 8 February 2017; Published 28 February 2017

Academic Editor: Saulius Juodkazis

Copyright (c) 2017 A. V. Yakunov et al. This is an open access article distributed under the Creative Commons Attribution License, which permits unrestricted use, distribution, and reproduction in any medium, provided the original work is properly cited.

\begin{abstract}
Raman scattering has been used to study the influence of $2.45 \mathrm{GHz}$ microwave on the structure of water. It has been shown that treatment of the distilled water samples by electromagnetic field leads to long-term changes in the vibrational density of states. It was established that the retention time of structural changes of the water samples depends on the sample volume. The experimental results have been interpreted on the basis of the percolation model. It has been suggested that the change in the chemical composition of the water treated by microwaves can lead to a change in the structure of the percolation cluster formed by the network of hydrogen bonds. The time of the equilibrium structure recovery of the percolation cluster after termination of the microwaves depends on the cluster size and is much slower than the recovery in the chemical composition of water.
\end{abstract}

\section{Introduction}

Electromagnetic waves in the microwave range corresponding to ultrahigh frequency (UHF) of $1-30 \mathrm{GHz}$ are widely used in various industrial processes at electronic, food, pharmaceutical, and chemical industry [1]. Microwaves are efficiently absorbed by a number of substances and therefore they are used primarily for fast and controlled heating.

At the same time, many researchers are paying attention to the fact that using the common convective type heater and a microwave oven at the same temperature increase may lead to different implications $[2,3]$. There are so-called specific effects of microwaves. The first of them, although they have a thermal nature, cannot be reproduced in the common convection heater. These are (a) the effect of overheating, (b) selective heating, (c) the temperature gradients on the border, and so forth [4].

The possibility of nonthermal effect of microwaves on the different processes and substances, despite the considerable amount of experimental work, is the subject of lively debate recently $[2,5,6]$. The mechanism of this influence is not entirely clear. The energy of single microwave photons (e.g., $3 \mathrm{GHz}$ corresponds to the energy of $10^{-3} \mathrm{eV}$ ) is several orders of magnitude smaller than the energy of chemical bonds. Therefore, a direct effect (like photochemical transformation) seems improbable. The ability of the alternating field to redirect the interacting polar molecules and to change the activation energy of a chemical reaction is considered as more realistic. Theoretically, there may be other mechanisms of nonthermal exposure, requiring, however, experimental approval [2].

Due to its fundamental and technological significance, the reports on the effect of microwaves on the properties of water and aqueous solutions, which serve as the working medium or mediator in many industrial processes and are an essential component of living organisms, are of particular interest.

The experimental investigations in which the nonthermal effects of microwaves have been registered can be divided into two classes. In the first case, the object under study is exposed with low-intensive waves when a thermal effect, even taking into account the "specific" influences, can be neglected. In particular, in [7], it was reported that the conductivity of the $\mathrm{NaCl}$ aqueous solution was changed at the result of the treatment of various microwaves with the frequency at $2.45 \mathrm{GHz}$. The amplitude of the electric field component was about $10^{4} \mathrm{~V} / \mathrm{m}$, which eliminates the heating of the sample more than $0.1^{\circ} \mathrm{C}$.

In the other type of experimental works, the nonthermal microwave effects have been observed simultaneously with 
heat or at least with their action but leaded to the significantly different results. The properties obtained under the influence of microwaves are commonly observed during long time, but they are absent in the case of conventional heating (so-called memory effect). The typical relaxation processes in aqueous media have a characteristic time of about picoseconds, and it remains unclear by what processes can be configured as slow dynamics.

The influence of microwaves on surface tension of the droplets of water and ethylene glycol was investigated in [8]. During the irradiation, a decrease in the predicted coefficient associated with heating (about $60^{\circ} \mathrm{C}$ ) has been observed for both samples. After the termination of irradiation, the surface tension of the ethylene glycol returned to its initial value at a rate typical for cooling. At the same time, the surface tension of water remained significantly below baseline for over 1 hour after the end of irradiation.

In [9], wherein the aqueous $\mathrm{KOH}$ solutions were used in the process of etching of the silicon surface, the concomitant effect of microwave at $2.45 \mathrm{GHz}$ on the properties of water was reported. During evaporation, the formation of larger crystals in the case when solutions were prepared from water heated by microwaves in comparison with the case of water warmed by conventional manner has been observed. The preservation of these properties is also noted in the long term (more than 20 days).

The authors of these papers do not specify the mechanism of interaction; however, they connected the observed effects with the change of water structure.

At the moment, an idea of the structure of water as a continuous network of hydrogen bonds, forming a single macroscopic cluster, is acknowledged. To describe the features of the vibrational spectra of disordered media, the ideas of fractal geometry and the theory of percolation are applied effectively [10]. According to the percolation model [11], water structure at room temperature can be represented as an infinite percolation cluster containing smaller clusters, small groups of molecules, and individual molecules in its cavities. Low-frequency $\left(1-100 \mathrm{~cm}^{-1}\right)$ Raman spectra provide the information about the structure of the pseudopolymer network of liquids with hydrogen bonds [12].

In this paper, the facts of long-term preservation of the structure of water under the influence of $2.45 \mathrm{GHz}$ microwaves have been checked using the low-frequency Raman spectroscopic monitoring.

\section{Low-Frequency Raman Spectrum of Water}

Raman spectrum of water is rather complicated and consists of a set of the bands with different intensities and widths. In the field of even more low frequencies $\left(<100 \mathrm{~cm}^{-1}\right)$, the Raman spectrum of water is hidden by intense wing of Rayleigh scattering. In order to find a useful unstructured signal, usually perform spectrum transformation [13]:

$$
I_{\text {red }}(\nu)=\frac{I(\nu) \cdot v}{n(\nu)+1}
$$

where $I_{\text {red }}(\nu)$ is the reduced spectral distribution of intensity; $n(\nu)=\left(e^{h v / k T}-1\right)^{-1}$ is Bose-Einstein factor. For the analysis of vibrational spectrum, the density of states function $g(\nu) \sim$ $I_{\text {red }}(\nu)$ is used.

At low frequencies, the homogeneous media and the crystals are characterized by a power law, that is, $g(v) \sim \nu^{\alpha}$, where $\alpha=2$, which corresponds to the classical Debye dispersion law. At the same time, for some liquids and amorphous substances, the exponent is less than 2 . Within the percolation model for partially ordered media, such deviation can be interpreted as a manifestation of an intermediate between acoustic and optical types of vibrational excitations. Since these excitations are localized in the lattice of the percolation fractal clusters, they are called "fractons" [14].

The measurements of low-frequency vibrational spectrum of water in the range of $0.1-50 \mathrm{~cm}^{-1}$ [15] indicate the existence of anomalies in the spectrum density at $1 \mathrm{~cm}^{-1}$. This frequency is called crossover frequency $v_{\text {co }}$ and corresponds to the transition from phonon $(\alpha=2)$ to fracton $(\alpha<2)$ section of the dispersion curve. In the presence of multiscale inhomogeneities, which are typical of fractal objects, it is directly related to the fractal dimensionality of the medium. The crossover frequency $v_{\text {co }}$ determines the maximum size of the heterogeneity of the percolation cluster in tetragonal network of hydrogen bonds, and for water this value is about $10 \mathrm{~nm}$ [15]. That is, according to the percolation model, the water should be considered as an inhomogeneous medium in the nanometer scale.

Experimentally, the parameter $\alpha$ can be determined from the spectral distribution of the Raman intensity, converted using formula (1). The density of states function $g(\nu)$, built in double logarithmic scale, on a portion of the spectrum will be a straight line with the slope equaling $\alpha$.

\section{Experimental Procedure}

We investigated the low-frequency Raman spectra in the region of $15-100 \mathrm{~cm}^{-1}$ of standard water for injection in glass ampoules of 2,5 , and $10 \mathrm{~mL}$ (conductivity $<1.1 \mu \mathrm{S} \cdot \mathrm{cm}^{-1}$, the dry residue concentration $<0.001 \%)$. Water in the unsealed ampoules was heated to boiling in a microwave oven with adjustable power of microwave radiation and, separately, on a hotplate. The operating parameters in each case have been chosen to provide the same heating time, $\sim 1 \mathrm{~min}$.

Subjected to single heating, samples were stored in a refrigerator and have been removed for short (about 20 minutes) time for sequential recording of 4-5 spectra at room temperature $\left(\sim 20^{\circ} \mathrm{C}\right)$. This number of records was chosen for the detection of possible trend. The first cycle of measurements was carried out in 1 hour after treatment. During this time, the water in all ampoules cooled to room temperature. One unsealed $5 \mathrm{~mL}$ ampoule was used as a control during the entire experiment for 10 days. The unsealed ampoules during all storage period were closed by caps (unhermetically) that prevent contamination.

Unpolarized Raman spectra of water have been recorded at automated double-grate spectrometer DFS-24. Spectra 
were excited by line at $514 \mathrm{~nm} \mathrm{Ar}^{+}$laser (output power $\sim 80 \mathrm{~mW}$ ). The focused by lens laser beam was passed vertically along the axis of the ampoule with water. The area has been focused on the entrance slit of a monochromator with an average middle slit about 200 microns. Each time before the main experiment, we tested the spectrometer detecting the signal from control sample (vacuum-sealed ampoule).

\section{Results}

In Figure 1(a), the Raman spectra of three samples (control water and water heated by convectional method and by microwaves in 1 hour after treatment) in the range of 15$100 \mathrm{~cm}^{-1}$ are shown.

Figure 1(b) demonstrates in a double logarithmic scale the density of states functions derived from the corresponding spectra by means of transformation (1).

It is seen that part of the spectrum in the range of 15$50 \mathrm{~cm}^{-1}$ is linear in a logarithmic scale; hence the dependence of the density of states on frequency is described by a power function $g(v) \sim v^{\alpha}$. The slope of the linear trends (coefficient $\alpha$ ) and standard error of the estimate for the control sample is $1.051 \pm 0.001,1.044 \pm 0.002$ for water heated by convectional method, and $1.123 \pm 0.002$ for microwave heating.

Figure 2 shows the results of measurements of the structural parameter $\alpha$ held within 10 days for water samples subjected to microwave irradiation and conventional heating. Each point on the plot corresponds to an average of 4-5 values of $\alpha$ resulting from several consecutive recordings of spectrum. The standard errors have been calculated for confidence level of 0.99 .

To visualize the error intervals reflecting the spread of data in each cycle of measurements, the points in the diagram are shifted slightly relative to each other along the timeline.

Previously [12], it was found that the structural parameter $\alpha$ depends on the temperature of both the sample and the environment, the method of preparation, and storage time of the investigated substance. In the long series of measurements for the same sample, the parameter fluctuations about $2 \%$ are detected. Its value is also influenced by the presence of dissolved air in nonpressurized water sample.

Figure 2 shows that, for the control sample, the fluctuations of parameter $\alpha$ do not go beyond the range of statistical error over a long time (10 days). Heated to boiling by convectional method and cooled to room temperature water is characterized by several large statistical spread of the primary data, but the parameter also does not extend beyond $2 \%$ of fluctuations.

Heated in a microwave oven to boiling and then cooled to room temperature samples are characterized by a statistically significant increase in the parameter $\alpha$ in comparison with control water and water heated in the usual manner. This difference persists for a long period of time, depending on the volume of the treated sample. The parameter $\alpha$ returns to the control value: sample with volume at $2 \mathrm{~mL}, 5$ days $(120 \mathrm{~h})$; $5 \mathrm{~mL}, 10$ days $(240 \mathrm{~h})$; and $10 \mathrm{~mL}$, after about 20 days.

\section{Discussion}

The results of our experiments show the influence of microwave frequency at $2.45 \mathrm{GHz}$ on the low-frequency spectrum of water.

It is well known that the Raman spectrum of water in a wide frequency range is very sensitive to temperature. In low-frequency spectral range [15], the frequency of crossover changes $v_{\mathrm{co}} \approx 1 \mathrm{~cm}^{-1}$ at $t=20^{\circ} \mathrm{C}$ to $v_{\mathrm{co}} \approx 3 \mathrm{~cm}^{-1}$ at $t=80^{\circ} \mathrm{C}$, indicating a corresponding reduction in the size of irregularities. In [12] it was noted that heating leads to the gradual destruction percolation cluster and at a temperature above $t=50^{\circ} \mathrm{C}$ in the low range of the function $g(v)$ ceases to obey a power law.

Our results suggest the existence of additional (not only caused by heating) perturbations introduced by microwaves and influencing the structure of water.

The structure of the hydrogen bond network and the fractal dimension of the percolation cluster may change as a result of the tetrahedral coordination disorders caused by the presence of defects, such as those associated with the change of the number of hydrogen bonds of the molecule or change in the local charge of the group of molecules.

The structural organization of oxygen and hydrogen atoms in a network of hydrogen bonds is described by the known Bernal-Fowler rules. According to these rules, (1) each oxygen atom has two nearest neighboring hydrogen atoms and (2) only one hydrogen atom is present on each $\mathrm{O}-\mathrm{O}$ linkage.

The dissociation of water molecules in the liquid water or autoprotolysis extending the scheme $2 \mathrm{H}_{2} \mathrm{O} \rightarrow \mathrm{OH}^{-}+$ $\mathrm{OH}_{3}{ }^{+}$leads to the presence of some equilibrium level of hydroxyl ions and hydronium ions. The appearance of $\mathrm{OH}^{-}$ and $\mathrm{OH}_{3}{ }^{+}$ions breaks rule (1), resulting in so-called ion defects in the structure of water. Their concentration depends on the temperature. At $T=300 \mathrm{~K}$, their equilibrium concentration is equal to $10^{-7} \mathrm{~mol} \cdot \mathrm{dm}^{-3}$, which corresponds to $\mathrm{pH}=7$. The temperature change, among other things, by absorption of microwaves, leads to corresponding change in the equilibrium concentration of the ion defects.

However, the microwaves could give further influence on the reaction of autoprotolysis by the reorienting of the interacting polar molecules and a corresponding change in the activation energy. This possibility is indicated by the results of [16], where low intensity microwave radiation led to a change in $\mathrm{pH}$.

There are also defects due to violation of rule (2). They are called the orientational defects: D-defect corresponds to two protons on the line $\mathrm{O}-\mathrm{O}$ and $\mathrm{L}$-defect corresponds to a lack of protons on the line O-O. The concentration of the orientational defects depends on the stoichiometric composition of the water which follows from rule (2). The ability of the stoichiometry to locally change under the influence of microwaves due to mechanochemical reactions of the radical dissociation of water is confirmed by the data of [17], which recorded the appearance of the trace amounts of hydrogen peroxide $\left(\mathrm{H}_{2} \mathrm{O}_{2}\right)$ in water.

Hydrogen peroxide, acting as an orientational defect, not only could modify the structure of water but also affect 


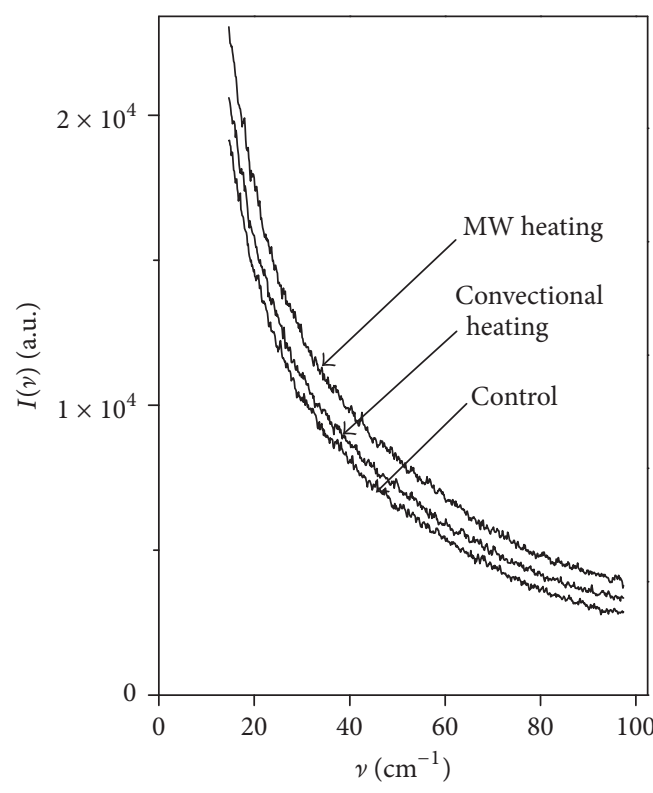

(a)

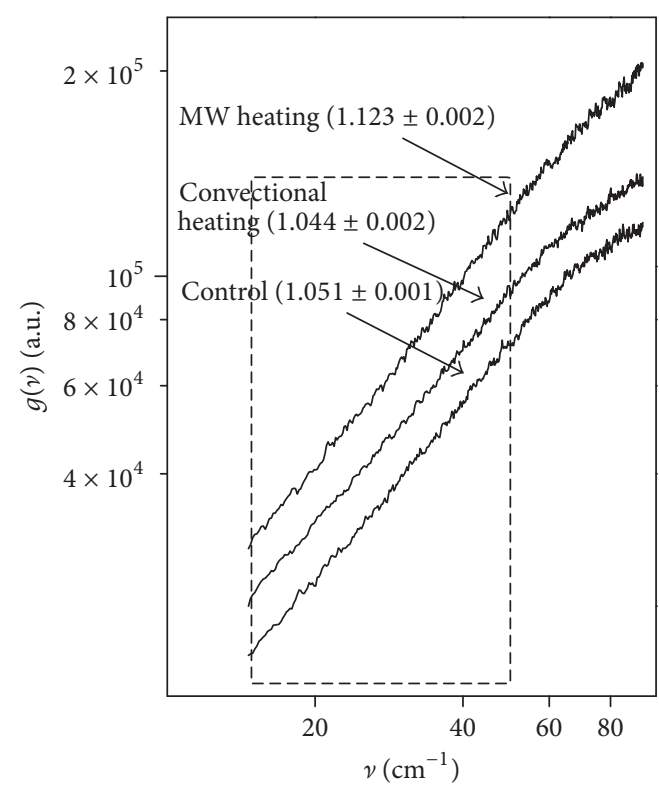

(b)

FIGURE 1: Raman spectra of three samples: control water and water heated by convectional method and by microwaves (a); the density of states functions of corresponding spectra (b). The slope and standard error of the estimate for each sample are shown in parentheses.

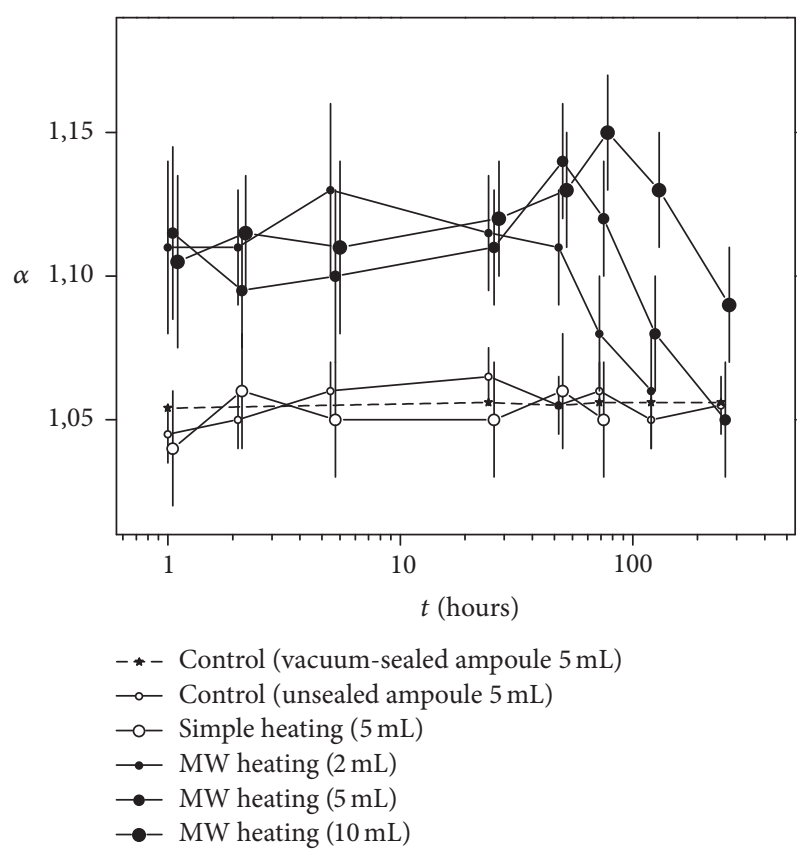

FIGURE 2: Dynamics of changes in the structural parameters of water samples within 10 days. The standard errors have been calculated for confidence level of 0.99 .

the slow relaxation processes. Whether this contribution is quite significant at extremely low concentrations so far is not known. Here, additional studies are needed.

In [12], it was shown that small additions of impurities significantly change the structure of the percolation cluster and, accordingly, its fractal dimension. The main source of impurities in our case is atmosphere. The influence of atmospheric gases on the low-frequency range of spectra of water contacting with the atmosphere is reflected as a small (about 2\%) increase of the structural parameter to some equilibrium value. The heating of water accelerates the diffusion process. When cooling, the concentration of atmospheric gas molecules is restored to equilibrium.

In our experiments, we compared the effect of microwave and convection heating to identical (from the same lot) samples of water, the content of the gases in which is negligible taking into account the technology of preparation. The vials were unsealed immediately before treatment. During the cooling process of water, the concentration of the atmospheric gas molecules due to diffusion increases to some equilibrium value.

Microwave treatment of water can lead to a change in the equilibrium concentration of impurities due to a specific effect of microwaves on the border "water-air," where a large temperature gradient occurs and hence the diffusion processes intensify [4].

Thus, the chemical composition of the initially clear water can markedly change after processing by microwaves compared with conventional heating.

The data of our experiments suggest that structural changes in the water treated by the microwaves are stored for several days and only then relax to their original settings. Moreover, the time of metastable state depends on the volume of the treated sample. It may be assumed that this dependence is due to the slower diffusion of impurities in a larger volume. However, the characteristic time for installing the equilibrium concentration would have the same order as the sample subjected to ordinary heat. In the last case, as follows from our experiments, the equilibrium is reached not later 
than 1 hour. Thus, the existence of a slow process of change of structure should be assumed.

In $[18,19]$, using cellular automata realized on a twodimensional square lattice, the authors modeled the dynamic processes in the water like a molecular and supramolecular level.

It was found that after pulse temperature perturbation the average number of hydrogen bonds per molecule relaxes to an equilibrium value regardless of the size of the system at a time about $10 \tau$. Here $\tau$ is a "quantum of time" of the cellular automata and is interpreted as the life time of the hydrogen bond ( $10 \mathrm{ps})$. At the same time, the fractal dimension of $2 \mathrm{D}$ cluster relaxes to equilibrium dimension with time constant $\tau_{F} \sim N^{1 / 2}$, where $N$ is the total number of elements (molecules) of the system. In the case of macrosystems with $N \gg \gg 1, \tau_{F}$ may have an arbitrarily large value, thereby ensuring infraslow dynamics of structural changes.

Thus, it can be assumed that the changes of the chemical composition of the water caused by the action of the microwaves lead to a change in its structure in macrovolume. Within the percolation model, this change is reflected as the increasing of the fractal dimension of the percolation cluster formed by hydrogen bonds and the corresponding increase of the exponent in the density of states function (structural factor). The time for establishing the equilibrium structure of the percolation cluster, after termination of the microwave, depends on its size and is much slower than the recovery in the chemical composition of water.

Note that the ultraslow dynamics of percolation cluster may underlie the mechanisms that form the so-called water memory: the long-term preservation of the properties is acquired as a result of any (usually weak) influences.

\section{Conclusions}

The obtained experimental data allow concluding the longterm changes of the structure of water after the microwave treatment. It is assumed that change in chemical composition of water treated by microwaves may lead to the changes of the percolation cluster formed network of hydrogen bonds. The recovery time of the equilibrium structure of the percolation cluster after termination of the microwave action depends on the cluster size and is much slower than the recovery of chemical composition of water.

\section{Competing Interests}

The authors declare that they have no competing interests.

\section{References}

[1] D. E. Clark and W. H. Sutton, "Microwave processing of materials," Annual Review of Materials Science, vol. 26, no. 1, pp. 299-331, 1996.

[2] J. Jacob, L. H. L. Chia, and F. Y. C. Boey, "Thermal and nonthermal interaction of microwave radiation with materials," Journal of Materials Science, vol. 30, no. 21, pp. 5321-5327, 1995.

[3] F. Langa, P. de la Cruz, A. de la Hoz, A. Díaz-Ortiz, and E. Díez-Barra, "Microwave irradiation: more than just a method for accelerating reactions," Contemporary Organic Synthesis, vol. 4, no. 5, pp. 373-386, 1997.

[4] C. Antonio and R. T. Deam, "Can 'microwave effects' be explained by enhanced diffusion?" Physical Chemistry Chemical Physics, vol. 9, no. 23, pp. 2976-2982, 2007.

[5] N. Kuhnert, "Microwave-assisted reactions in organic synthesis-are there any nonthermal microwave effects?" Angewandte Chemie-International Edition, vol. 41, no. 11, pp. 1863-1866, 2002.

[6] C. O. Kappe, B. Pieber, and D. Dallinger, "Microwave effects in organic synthesis: myth or reality?" Angewandte Chemie, vol. 52, no. 4, pp. 1088-1094, 2013.

[7] K. Huang, X. Yang, W. Hua, G. Jia, and L. Yang, "Experimental evidence of a microwave non-thermal effect in electrolyte aqueous solutions," New Journal of Chemistry, vol. 33, no. 7, pp. 1486-1489, 2009.

[8] H. Parmar, M. Asada, Y. Kanazawa et al., "Influence of microwaves on the water surface tension," Langmuir, vol. 30, no. 33, pp. 9875-9879, 2014.

[9] R. Walczak and J. Dziuban, "“Microwave memory effect” of activated water and aqueous $\mathrm{KOH}$ solution," in Proceedings of the 15th International Conference on Microwaves, Radar and Wireless Communications (MIKON '04), vol. 1, pp. 253-256, Warsaw, Poland, May 2004.

[10] T. DeSimone, S. Demoulini, and R. M. Stratt, "A theory of percolation in liquids," The Journal of Chemical Physics, vol. 85, no. 1, pp. 391-400, 1986.

[11] A. Geiger, F. H. Stillinger, and A. Rahman, "Aspects of the percolation process for hydrogen-bond networks in water," The Journal of Chemical Physics, vol. 70, no. 9, pp. 4185-4193, 1979.

[12] N. Kuzkova, A. Yakunov, and M. Bilyi, "Low-frequency Raman spectroscopic monitoring of supramolecular structure in $\mathrm{H}$ bonded liquids," Advances in Optical Technologies, vol. 2014, Article ID 798632, 7 pages, 2014.

[13] R. Shuker and R. W. Gammon, "Raman-scattering selectionrule breaking and the density of states in amorphous materials," Physical Review Letters, vol. 25, no. 4, pp. 222-225, 1970.

[14] S. Alexander and R. Orbach, "Density of states on fractals: fractons," Journal de Physique Lettres, vol. 43, no. 17, pp. 625631, 1982.

[15] D. Majolino, F. Mallamace, P. Migliardo, F. Aliotta, N. Micali, and C. Vasi, "Spectral evidence of connected structures in liquid water: effective Raman density of vibrational states," Physical Review E, vol. 47, no. 4, pp. 2669-2675, 1993.

[16] G. Morariu, M. Miron, A. M. Mita, and L. V. Stan, "Microwaves electromagnetic field influence on $\mathrm{pH}$. Theoretical and experimental results," Review of the Air Force Academy, no. 1, pp. 4548, 2009.

[17] V. L. Vaks, G. A. Domrachev, Y. L. Rodygin, D. A. Selivanovskii, and E. I. Spivak, "Dissociation of water by microwave radiation," Radiophysics and Quantum Electronics, vol. 37, no. 1, pp. 85-88, 1994.

[18] L. B. Kier and C.-K. Cheng, "A cellular automata model of water," Journal of Chemical Information and Computer Sciences, vol. 34, no. 3, pp. 647-652, 1994.

[19] A. Yakunov and P. Yakunov, "Slow dynamics of water structure in cellular automata model," in Proceedings of the International Conference "Physics of Liquid Matter: Modern Problems", p. 140, 2004. 


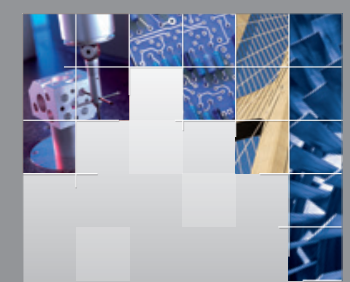

\section{Enfincering}
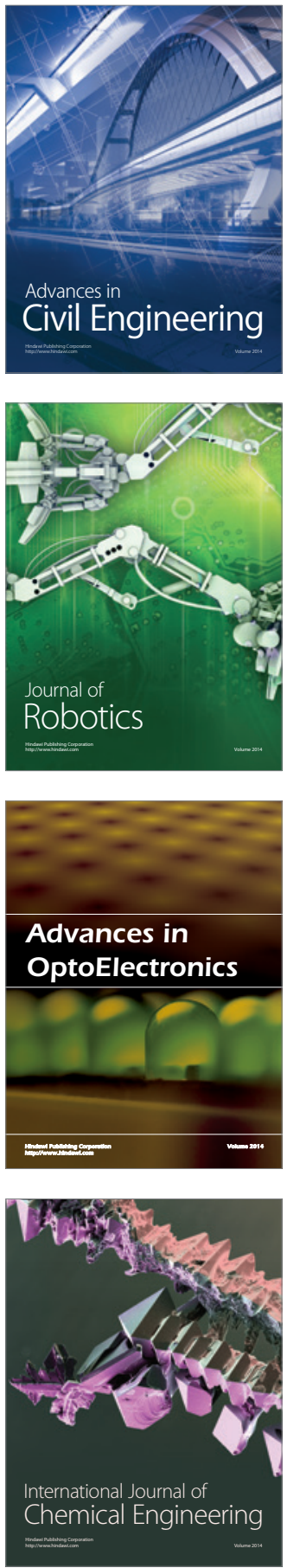

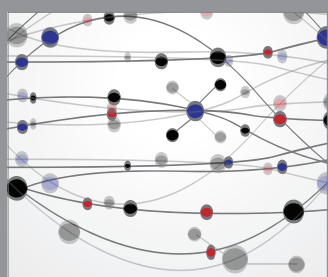

The Scientific World Journal

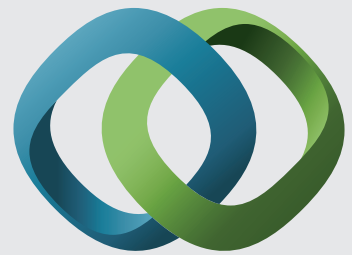

\section{Hindawi}

Submit your manuscripts at

https://www.hindawi.com
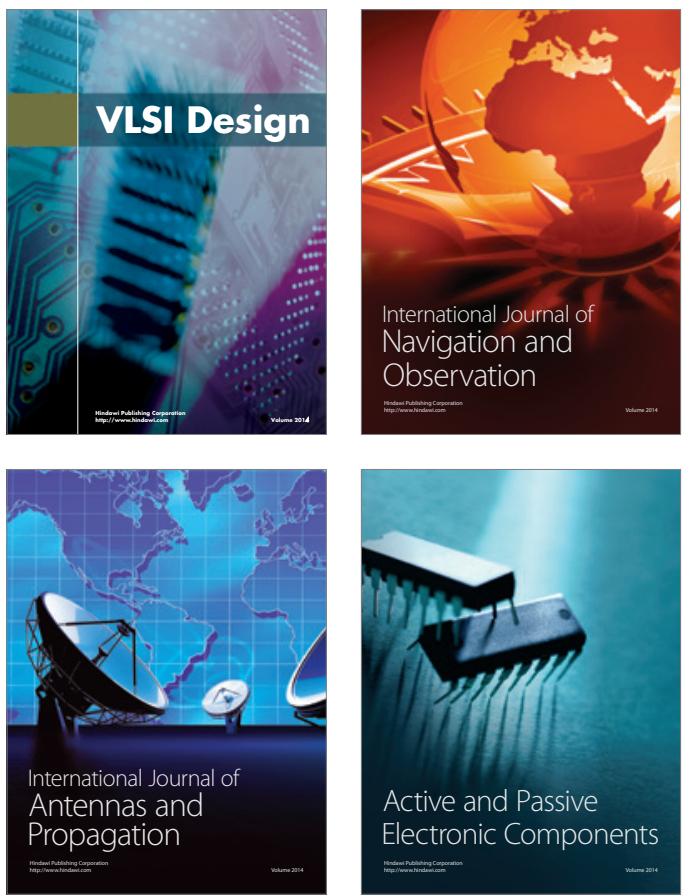
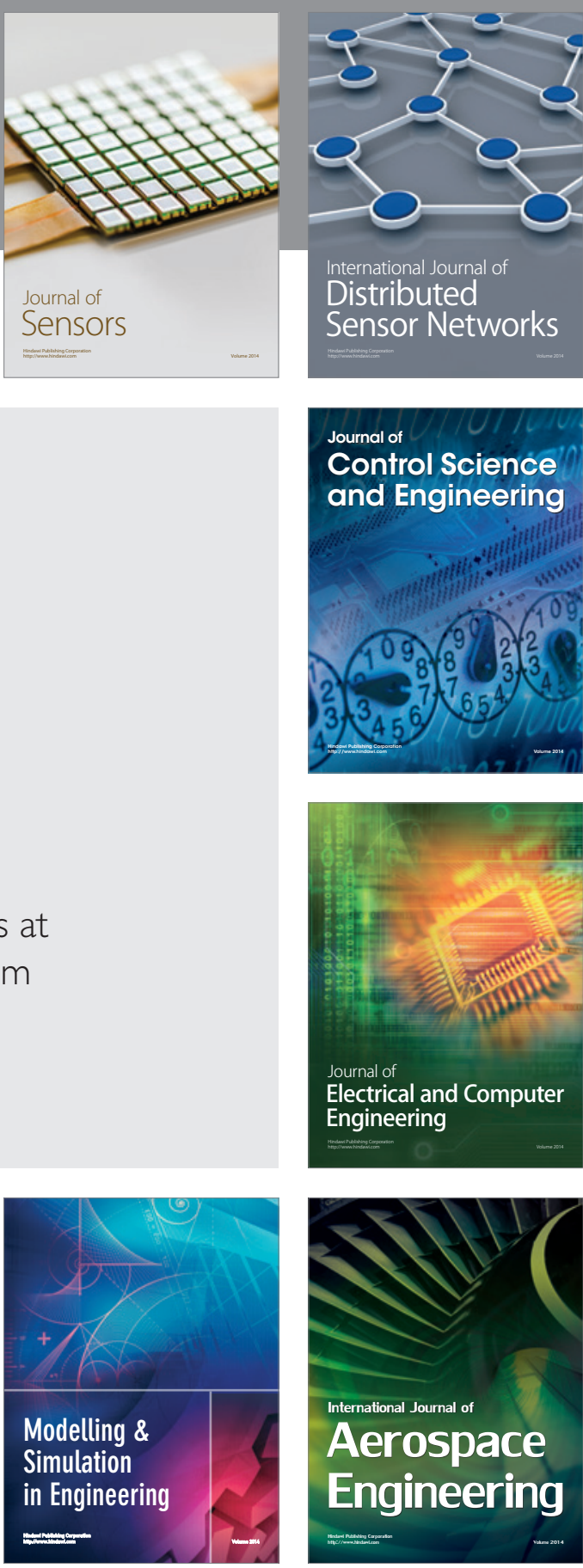

International Journal of

Distributed

Sensor Networks

$-$

Joumal of

Control Science

and Engineering
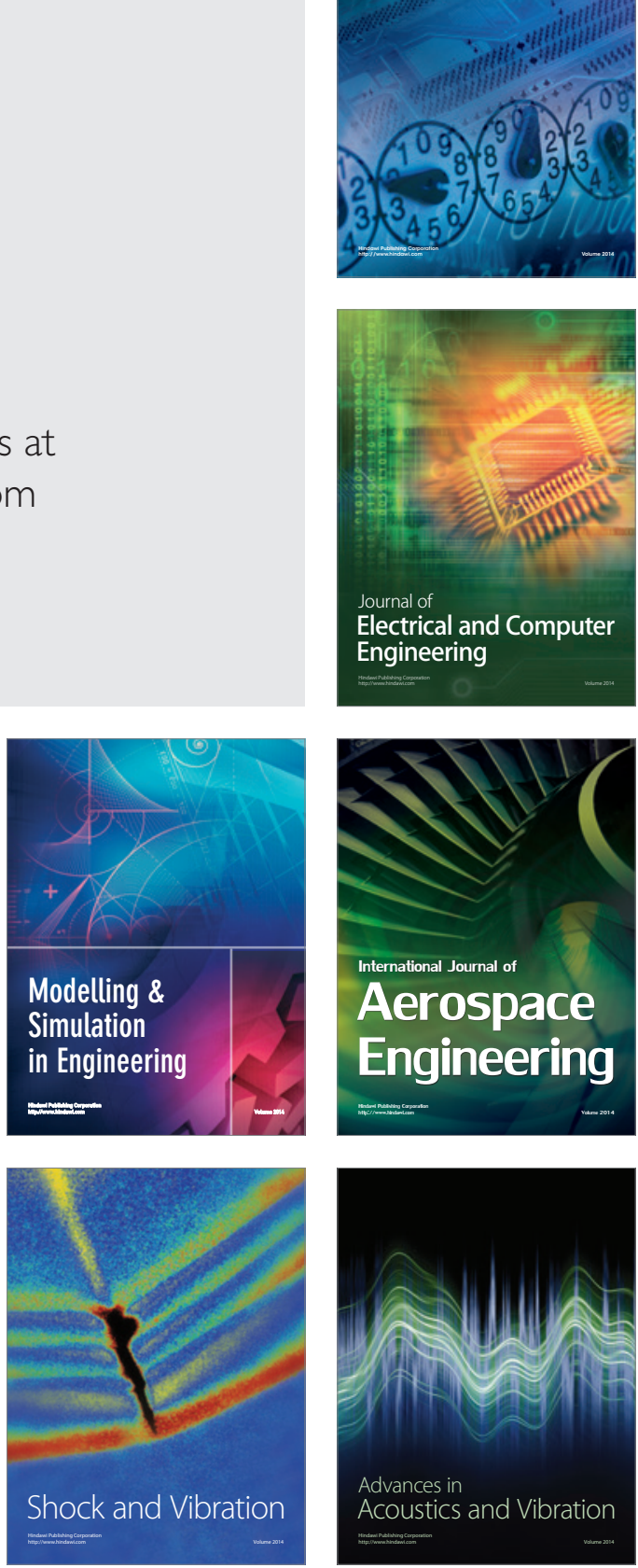